\title{
Civilisations
}

Revue internationale d'anthropologie et de sciences

humaines

$41 \mid 1993$

Mélanges Pierre Salmon II

\section{Les juifs marocains dans l'Archipel des Acores - début d'une nouvelle mentalité commerciale : l'exemple des Bensaude}

\author{
Fatima Sequeira Dias \\ Traducteur : Joao Gabriell Santana
}

\section{OpenEdition}

\section{Journals}

Édition électronique

URL : http://journals.openedition.org/civilisations/1727

DOI : $10.4000 /$ civilisations. 1727

ISSN : 2032-0442

\section{Éditeur}

Institut de sociologie de l'Université Libre de Bruxelles

Édition imprimée

Date de publication : 1 septembre 1993

Pagination : $403-413$

ISBN : 2-87263-094-5

ISSN : 0009-8140

\section{Référence électronique}

Fatima Sequeira Dias, « Les juifs marocains dans l'Archipel des Acores - début d'une nouvelle mentalité commerciale : l'exemple des Bensaude », Civilisations [En ligne], 41 | 1993, mis en ligne le 30 juillet 2009, consulté le 30 avril 2019. URL : http://journals.openedition.org/civilisations/1727 ; DOI : 10.4000/civilisations. 1727

Ce document a été généré automatiquement le 30 avril 2019.

(c) Tous droits réservés 


\title{
Les juifs marocains dans l'Archipel des Acores - début d'une nouvelle mentalité commerciale : l'exemple des Bensaude
}

\author{
Fatima Sequeira Dias \\ Traduction : Joao Gabriell Santana
}

Je remercie Joao Gabriell Santana pour la traduction de ce texte.

1 De par sa localisation géographique, l'archipel des Açores est devenu le point d'arrêt obligatoire des voiliers qui croisaient dans l'Atlantique à partir du XV ème siècle. Offrant des ports d'abri aux flottes du Brésil et des Indes occidentales et orientales, à l'aller et surtout au retour, toutes les îles de l'archipel sont devenues des lieux de convoîtise pour tous ceux qui avaient été exclus du partage à la suite du traité de Tordesillas. Les diverses îles sont ainsi devenues la cible de nombreuses attaques des pirates et des corsaires français, anglais, hollandais et "algériens".

2 Malgré les attaques fréquentes des pirates, le Portugal, puissance responsable du peuplement de ces îles à partir de 1436, est parvenu à assurer, plus ou moins efficacement selon les époques, les fonctions qui lui avaient été assignées, à savoir, servir de points d'escale et de repos aux flottes des Indes ${ }^{1}$, et constituer en même temps des communautés agricoles dont la vocation était l'approvisionnement en céréales du royaume et des places fortes portugaises du Nord de l'Afrique.

3 Ainsi, dès l'aube des découvertes maritimes portugaises, l'archipel des Açores a été lié aux circuits de navigation et de commerce existants, en approvisionnant les vaisseaux et les caravelles, puis les navires à vapeur, et de nos jours, une grande partie de la navigation aéronautique de l'Atlantique nord, restant lié et intégré à l'économie de marché apparue avec l'époque moderne.

4 Localisé à mi-chemin de grands centres économiques tels Amsterdam, Londres ou New York depuis le XVII ème jusqu'au début du XX ${ }^{\text {ème }}$ siècle, l'archipel a toujours contribué à l'approvisionnement agricole de ces centres et a répondu aux sollicitations de la demande 
avec de nouvelles productions vouées au déclin lorsque cette demande venait à diminuer d'importance. Ainsi, dans une succession continue de "cycles" économiques basés sur les productions agricoles, (on ignore encore l'existence de minerais dans le sous-sol), l'archipel a produit tout au long des époques, en régime de substitution, du pastel, du vin, du blé, du maïs, des oranges et, de nos jours, du bétail bovin².

Néanmoins, le régime de monoculture n'a jamais été complètement introduit notamment parce que la demande de la métropole qui se trouvent également à mi-chemin des centres économiques, n'a jamais coïncidé avec la demande internationale des produits agricoles de l'archipel. Par conséquent, l'archipel, doublement dépendant, était obligé de développer une certaine alternance des cultures en vue de répondre à deux types différenciés de demande agricole.

6 Cependant les cultures dites coloniales n'ont jamais été introduites dans l'archipel du fait de la douceur du climat et de leur faible rentabilité au vu de l'exiguïté du territoire. Elles ont contribué de ce fait à ce que le binôme "esclavage noir - production de type colonial" ait été absent du parcours économique de l'archipel. La quasi totalité de la population de l'archipel était d'origine portugaise avec néanmoins des éléments d'origine flamande et française ${ }^{3}$. Les quelques esclaves existants dans la société insulaire étaient, par conséquent, occupés aux travaux domestiques, comme c'était le cas dans le royaume.

7 Toutefois, la pauvreté de ces îles, comme en témoigne la redistribution déficiente des terres, aggravée par la succession de calamités physiques qui dévastaient fréquemment l'archipel - tremblements de terre et volcans -, a contribué à ce qu'il y ait eu une émigration importante des habitants dès le début du peuplement.

8 Ainsi, malgré le fait que l'archipel des Açores était intégré dans les circuits de navigation Atlantique et qu'il essayait de répondre aux sollicitations d'une demande extérieure, nous percevons dans son intégration économique restée partielle jusqu'à la moitié du XIX ${ }^{\mathrm{e}}$ siècle, des phénomènes de désarticulation, de dualisme et de développement extraverti vis-à-vis des marchés exogènes (métropole et pays du centre), mais aussi à l'intérieur de chaque île et entre les îles privilégiées et le reste de l'archipel ${ }^{4}$.

9 La proximité du continent africain, des archipels portugais de Madère et du Cap Vert et des îles espagnoles des Canaries, a amené les géographes européens jusqu'à la fin du siècle dernier à appeler toutes ces îles, y compris l'archipel des Açores, "îles d'Afrique" alors que le climat de l'archipel des Açores et ses productions agricoles sont de type tempéré atlantique et donc différent de ceux des autres archipels mentionnés.

Toutefois, cette "proximité" relative de l'Afrique et la croyance en un développement économique "extraordinaire" dû à son intégration dans l'économie Atlantique dominée alors par le Royaume Uni ont, sans l'ombre d'un doute, contribué à attirer une importante communauté juive originaire du Maroc, au début du XIX ${ }^{\mathrm{e}}$ siècle.

Il faut néanmoins signaler que les Juifs marocains en fuite se sont dirigés non seulement vers l'archipel des Açores, mais aussi vers l'Algarve, le sud de l'Espagne et Gibraltar ${ }^{6}$. Les documents mentionnent leur établissement dans toutes ces régions vers la même époque et font état du fait que leur activité économique principale était le commerce ambulant à petite échelle.

12 Si ces commerçants juifs, en se dirigeant vers des marchés organisés, s'intègrent et se diluent dans le tissu économique existant, il n'en va pas de même aux Açores où leur influence et leur nouvelles méthodes commerciales nous amènent à affirmer qu'ils sont 
devenus la pièce maitresse du commerce international, contribuant de manière décisive au développement de l'intégration économique de l'archipel.

Leur installation avait un caractère provisoire comme nous le confirme le fait que les hommes arrivaient en premier lieu, faisant venir plus tard leurs épouses et leurs enfants. Certains d'entre eux, avant de s'établir définitivement dans l'archipel, avaient séjourné provisoirement dans la métropole, ce qui nous amène à penser que leur départ vers l'archipel était également envisagé comme transitoire. D'ailleurs, quelques uns des juifs qui ont séjourné aux Açores ont fini par émigrer vers le Brésil ${ }^{7}$.

Dès le début de leur peuplement, les Açores ont toujours attiré de nombreux étrangers qui, tirant parti de "la supériorité que leur donnaient l'information, l'intelligence et la culture..." ${ }^{\prime 8}$, se sont emparés du commerce d'import-export. Dans ce sens, la question importante est de savoir dans quelle mesure les commerçants juifs vont perturber les pratiques commerciales existantes dans l'archipel, et par là même, menacer les intérêts de la communauté marchande anglaise résidente dans ces îles9 .

'arrivée des juifs aux Açores se situe entre fin 1818 et début 1819; les deux registres mentionnant leur arrivée à l'île de $\mathrm{S}$. Miguel, ne s'accordent pas entre eux ${ }^{10}$.

16 Néanmoins, dès le deuxième semestre de 1820 , on commence à spéculer sur les gains spectaculaires obtenus par leurs ventes ambulantes ${ }^{11}$, ce qui leur a valu d'être la cible des commerçants locaux qui feront tout pour mettre un terme à cette concurrence néfaste.

17 Malgré le fait que l'implantation de ces juifs ait eu lieu par vagues successives, Abraao Bensaude, Salao Buzaglo, Aarao Benayon, Jacob Matana, Isaac Sentob et Aarao Aflalo furent les pionniers de cette émigration ${ }^{12}$.

18 Après l'établissement de ces pionniers, des incidents eurent lieu entre les commerçants locaux et ces nouveaux-venus. Néanmoins, la mise en place d'un régime libéral au Portugal à contribuer à ce que l'on leur ait permis de s'établir dans ces îles (S. Miguel 1821 et Terceira 1831).

19 Le succès de ces commerçants juifs est basé sur une politique de rotation accélérée des stocks, ce qui a créé des mécanismes économiques et financiers novateurs dans le marché insulaire. En s'infiltrant partout dans l'île, parcourant les lieux les plus éloignés..., vendant aux prix les plus bas, octroyant des facilités de crédit et offrant une plus grande variété de tissus, ces commerçants contribueront ainsi au "redimensionnement du marché à travers la généralisation de nouvelles concurrences dans le sens d'une plus grande libéralisation des prix", comme l'affirme David Justino lorsqu'il identifie le même phénomène dans la province de l'Algarve au début des années vingt ${ }^{13}$.

20 Cependant, les difficultés rencontrées par les premiers commerçants juifs des Açores allaient continuer pendant de nombreuses années avec des accusations fréquentes de concurrence déloyale. Ils étaient également rendus responsables de la fuite de la monnaie locale ${ }^{14}$.

21 La seule émeute connue contre des individus et des biens d'origine hébraïque, date de 1834 et eut lieu dans la ville de Ponta Delgada.

22 Toutefois, les émeutiers furent rapidement dissuadés de pratiquer de tels actes de vandalisme car, comme beaucoup de juifs possédaient la nationalité britannique, les autorités locales craignaient des représailles de la part du gouvernement anglais ${ }^{15}$. En outre, comme la Grande-Bretagne était le principal marché de destination des oranges de 
S. Miguel, il ne convenait pas de mettre en péril les importants intérêts économiques locaux avec des actions imprudentes.

Dans la seconde moitié du XIX siècle, on constate que les sentiments d'animosité vis-àvis de la minorité juive ont disparu et on peut affirmer que les juifs de l'île de S. Miguel étaient déjà intégrés dans le tissu social des Açores. Malgré le fait que la pratique de mariages mixtes n'était pas fréquente ${ }^{16}$, les liens de sociabilité sont devenus nombreux ${ }^{17}$. La communauté israélite de l'île Terceira se trouvait également intégrée dans la société locale, comme en témoigne le fait qu'elle a contribué activement à la cause libérale notamment avec de l'argent et des lits pour l'armée casernée dans l'île avant le débarquement du Mindelo ${ }^{18}$. Le caractère cosmopolite de l'île de Faial a aussi contribué à l'intégration sociale rapide des juifs qui s'y étaient installés ${ }^{19}$.

Il importe néanmoins de mentionner que la caractéristique dominante de cette communauté était la constante mobilité, visible dans le changement fréquent de résidence, dans les voyages périodiques vers les autres îles de l'archipel et à l'étranger, dans les nombreuses fois qu'ils signaient des écritures avec des tiers dans les divers endroits parcourus. Cette mobilité, source de maintien d'un réseau profond de liens et de contacts avec l'extérieur, caractérisait et identifiait la communauté juive par rapport à la communauté locale, fermée sur elle-même.

Le premier registre douanier mentionnant des importations effectuées par un commerçant juif est celui relatif à Salao Buzaglo qui, le 12 décembre 1821, a enregistré à la douane de Ponta Delgada des marchandises d'une valeur supérieure à $4.500 \$$ arrivées par la chaloupe anglaise "Sector" provenant de Gibraltar ${ }^{20}$. Dans les années suivantes, on assiste à de nombreuses importations effectuées par divers commerçants juifs. Ce n'est que dans les années trente que, Salomao Bensaude, en association avec son cousin Elias, est devenu prospère - aspect révélateur de ce que la concurrence affectait non seulement les "commerçants établis" mais aussi les "commerçants nouveaux-arrivés" et de ce que la "chance" dans les affaires finissait par être contraire si elle n'était pas associée à une gestion adéquate. Une hiérarchie commerciale s'esquissait également entre les commerçants juifs, mais, semble-t-il, favorable uniquement aux Bensaude.

La documentation consultée nous permet d'affirmer que tous les juifs installés dans ces îles se consacraient au secteur commercial.

Après la construction du port maritime de Ponta Delgada, à partir de 1861, les principaux investissements dans les secteurs secondaire et tertiaire appartiennent en majorité à des capitaux juifs et en quasi-monopole au groupe "Bensaïde et C. Ldt". Cette situation ne sera toutefois acquise qu'à la fin du siècle dernier quand le nombre de familles d'ascendance hébraïque aura diminué radicalement dans l'archipel, après les pénibles reconversions économiques imposées par la crise dans la production des oranges. Le nombre de familles juives sera alors inférieur à la dizaine, éparpillées dans tout l'archipel, alors qu'il avait été proche des deux cents vers le milieu du XIX ${ }^{e}$ siècle.

Aujourd'hui restent uniquement trois noms de famille révélateurs de leur ascendance Adrahi, Bensaude et Delmar. La famille Bensaude continue à jouer un rôle économique fondamental au sein de la société açorienne.

29 La diversité des affaires des cousins Bensaude - vente de marchandises importées, achat et exportation de produits agricoles régionaux, escompte de lettres de change, transferts et change de monnaies étrangères, investissement de capitaux dans des immeubles urbains et ruraux, etc. - explique les raisons du succès du groupe. 
urs, à travers la consommation, les cousins Bensaude vont esquisser la première tentative d'intégration économique de l'archipel. Ils ont stimulé les échanges inter-îles, tantôt comme activité de compensation en vue de dépasser les préjugés des autochtones en matière de fuite des devises, tantôt comme recherche de biens susceptibles de procurer des meilleures plus-values en dehors du marché insulaire, et ont été à la base de l'augmentation de ces échanges jusqu'aux années cinquante/soixante du siècle dernier, moment où cette tendance s'infléchit. La diminution progressive de la complémentarité des productions agricoles régionales (chute du prix des céréales dans le marché portugais, destruction des vignes par l'action du phylloxera, détérioration de la qualité des oranges due à des maladies diverses) et l'absence d'une stratégie basée sur une répartition régionale du travail, ont fini par accentuer la dépendance des îles plus petites vis-à-vis des capitales de districts et par confiner l'archipel à trois îles uniquement: S. Miguel, Faial e Terceira.

31 En conclusion, la réussite commerciale des Juifs aux Açores a été due à une plus grande agressivité dans les affaires, agressivité démontrée toujours par les commerçants "nouveaux-arrivés". En outre, la pratique d'une culture différente en ce qui concerne les affaires, associée à la manipulation ancestrale de la monnaie et des lettres de change, à l'utilisation d'une comptabilité sophistiquée, au manque de préjugés concernant la vente en gros et au porte-à-porte, le fait de ne pas être affectés par les contrariétés des longs voyages ou les ventes à crédit, ainsi que le réseau complexe de liaisons et de contacts commerciaux et familiaux, tous ces facteurs ont contribué à l'installation rapide de ces Juifs dans l'archipel pendant le XIX ${ }^{e}$ siècle. Cependant, le succès commercial ne s'est pas maintenu car la concurrence était considérable et les commerçants locaux ont commencé à adopter les pratiques commerciales caractéristiques des Juifs. D'ailleurs, à longue échéance, les entreprises juives ont été celles qui ont eu la durée de vie la plus courte à l'exception de l'exemple paradigmatique des Bensaude.

Les Bensaude, par les ramifications généalogiques de Abraao et de son fils José, de Elias et de ses fils Henrique et Walter, et de Salomao et de ses fils Abraao et Salom, furent les pionniers des principaux investissements dans l'industrie des Açores (tabac, ananas, alcool et betterave sucrière), dans les banques (Banco Comercial de Lisboa e Açores et plus tard à partir de 1912, Banco Micaelense), dans les assurances (Companhia Açoreana de Seguros), dans les transports maritimes et aériens (Empresa de Navegaçao Açoreana e Sociedate Açoreana de Transportes Aéreos), dans le tourisme (Sociedade Terra Nostra). Ils sont ainsi devenus un des principaux groupes économiques portugais au niveau national pendant les premières décennies du $\mathrm{XX}^{\mathrm{e}}$ siècle.

33 Les nationalisations économiques portugaises dans la période révolutionnaire de 1974/75 ont affecté profondément le groupe Bensaude. Par conséquent, il est difficile de prévoir ce que sera son comportement futur par rapport à la pleine intégration du Portugal dans la Communauté européenne d'après 1992. 


\section{ANNEXES}

\section{Abréviations}

B.P.A.P.D. - Bibliothèque publique et archive de Ponta Delgada

A.N.T.T. - Archive Nationale de la Torre du Tombo

B.P.A.A.H. - bibliothèque publique et archive d'Angra do Heroismo

B.P.A.H. - Bibliothèque publique et archive de Horta

A.C.C. - Archive de la Chambre de Commerce.

\section{NOTES}

1. Joao Marinho dos Santos, Os Açores nos secs. XV $\mathrm{XVI}$, Colecçao fontes para a historia dos Açores, Secretaria Regional de Assuntos Culturals, s/d, 2 vols.

2. Carreiro da Costa, Esboco historico dos Açores, Ponta Delgada, I.U.A., 1978, pp. 185-ss.

3. Ibidem, pp. 53-87.

4. José Paulo Martins Casaca, "Economia insular e economia arquipelagica-Uma confrontaçao necessaria" in Arquipélago, Economia, $n^{\circ}$ 1, Revista da Universidade dos Açores, 1988, pp. 61-74.

5. Azevac, Iles d'Afrique, Paris, Fermin Didot, frères éditeurs, 1848 et Ardoin et autres, Dictionnaire universel du commerce de la banque, Bruxelles, Ed. Lacroosse, 1840, 2 ed., 2 vols.

6. José Maria de Abecassis, Genealogia hebraica, Portugal, e Gibraltar, sécs. XVII a XX, Lisboa, Liva Ferin, 1990, 5 vols.

7. Notamment les héritiers de Mery Sabat, Cf. : Fatima Sequeira Dias, "Moisés Sabat - um caso de insucesso na comunidade hebraica de Ponta Delgada no século XIX (...-1864) in arquipélago, Série Historia, Revista da Universidade dos Açores, vol. XI, 1989, pp. 195-231 et Joaquim Bensaude, Cf. : Alfredo Bensaude, A vida de José Bensaude, Porto, Litografia Nacional, 1936, p. 64.

8. Fernand Braudel, A dinamica do capitalismo, Lisboa, Teorema, 1985, p. 63 et La civilisation matérielle, économique et capitalisme, XV-XVIII ${ }^{\mathrm{e}}$ siècle, Paris, ARmand Colin, 1979, vol. III, p. 29.

9. Nestor de Sousa, "Sinais de presença britanica na vida açoreana (séculos XVI-XIX)" in Arquipélago, Revista da Universidade dos Açores, $n^{\circ}$ especial, Relaçoes Gra Bretanha, 1988, pp. 25-100.

10. L'inscription tumulaire de Abraham Bensaude au Cimetière israélien de Ponta Delgada fait référence à son arrivée à Sao Miguel en 1818, alors que les archives de la mairie, datées du début des années vingt réfèrent "les commerçants "Hebreos" arrivés il y a quelques mois...", Cf. : B.P.A.P.D., Libro de Registos da Camara de Ponta Delgada, 1816-1823, fils 175-v a 176-v.

11. Ibidem, fls, 182 182-v.

12. Ibidem, fls $159-\mathrm{v}$ a $164-\mathrm{v}$.

13. David Justino, A formaçao do espaco economico nacional-Portugal 1810-1913, Lisboa, Vega, 1989, vol. 1, p. 380. 
14. B.P.A.H. - Livro de Registos da Camara Municipal da Horta, $\mathrm{n}^{\circ}$ 14, fls 98 (lettre du 2 mai 1835), Consultas das juntas geraes dos districtos Administrativos do reino e ilhas Adjacentes do anno de 1848, Lisboa, I.N. 1849, districto da Horta, pp. 2-3.

15. B.P.A.P.D., Arquivo do Governo Civil de Ponta Delgada, Copiador de Correspondencia do... as autoridades eclesiasticas, 1833-1842, $\mathrm{n}^{\circ} 141$.

16. Mariage de la fille d'Abraham Bensaude en 1854 avec José Maria do Couto Severim, un catholique, cf. : Alfredo Bensaude, A vida de José Bensaude, Porto, op.cit., p. 63.

17. Quelques commerçants juifs étaient membres d'associations locales, notamment le clube Michaelense et l'Ateneu Commercial, et aussi étaient associés de l'organisation commerciale de la ville de Ponta Delgada, Cf. : A.C.C. - Livro de Correspondencia expedida, 1841-1857, fls 5-6-v et encore les commerçants juifs résidents à Ponta Delgada ont fait des dons aux victimes d'innombrables catastrophes, et ont aidé à financer l'édification de la Bibliothèque publique de Ponta Delgada, cf. : O Açoreano Oriental, n 331, le 21 août 1841, nº 384 le 27 août 1842 et le $n^{\circ} 385$ le 8 septembre 1842 .

18. A.N.T.T. - M.R. Maço 259-260, Provincia dos Açores, Comarca de Angra.

19. Marcelino de Lima "Os Judeus na ilha do Faial" in Boletimm do nucleo cultural da Horta, $\mathrm{n}^{\circ} 1$, vol. 1, décembre 1956.

20. B.P.A.P.D., Fundo Ernesto do Canto, Livro de Direitos de $15 \%$ cobrados na Alfandega de Ponta Delgada, $1821, \mathrm{n}^{\circ} 10$, fls 50-v-ss.

\section{RÉSUMÉS}

The arrival of the Jews in the Azores took place between the end of 1818 and the beginning of 1819. From the 1830's onwards their commercial success was starling due to a greater commercial aggressivity. This commercial success was linked to their ability to expand new lines of business, their experience in handling money, bills of exchange and other sophisticated accounting methods. Neither were they put off by long journeys, nor by selling on credit and their complex network of business contacts all contributed to the rapid settling of Jews in the archipelago in the $19^{\text {th }}$ century. Although they were not resented by the local population, this commercial success, with the exception of the Bensaude family, was relatively short-lived due to stiff local competition and led to the departure of the few remaining merchants from the archipelago after the second world war.

\section{AUTEURS}

\section{FATIMA SEQUEIRA DIAS}

Université des Açores - Ponta Delgada - Portugal 